\title{
DECOMPOSITIONS OF A LOOP INTO CHARACTERISTIC FREE SUMMANDS
}

\author{
GRACE E. BATES
}

It is known ${ }^{1}$ that if the group $G$ is the free sum of proper subgroups $G_{1}$ and $G_{2}$ (or free product for multiplicative groups), then neither $G_{1}$ nor $G_{2}$ is normal in $G$. Clearly, then, there is no nontrivial decomposition of a group into characteristic free summands. In this paper we show that the above statement on normality holds for free summands of a loop, but that it is possible, nevertheless, with relatively few restrictions, to obtain decompositions of loops into characteristic free summands. $^{2}$

We use definitions and theorems from a previous paper, ${ }^{3}$ referred to throughout as [I], but the statements of these are here repeated for the convenience of the reader.

Notations. If $J, K$ are subsets of a loop $L$, we denote by $J \cup K$ the set-theoretical sum of elements in $J$ and $K$; by $J \cap K$ the crosscut of $J$ and $K$; and by $J+K$ the subloop of $L$ which is generated by $J \cup K$. If $\alpha$ is a single-valued map of the subset $J$ of $L$ into some loop $T$, we denote by $J \alpha$ the image of $J$ under $\alpha$, and by $x \alpha$, the image of any element $x$ in $J$.

A half-loop $J$ is a set of elements with a rule of combination, + , defined for some ordered pairs of elements $(a, b)$ of $J$, and subject to the following three conditions:

i. If $a+b=c$ and $a+b=d$, then $c=d$.

ii. If $x+a=y+a$, then $x=y$; if $b+x=b+y$, then $x=y$.

iii. There exists an element, 0 , in $J$, such that $0+x=x+0=x$, for every $x$ in $J$.

A loop $L$ is a half-loop satisfying the added condition: If any two of the three elements $a, b, c$, are in $L$, then $a+b=c$ determines the third as an element of $L$. Every subset of a $\operatorname{loop} L$ which contains the 0 -element of $L$ is a half-loop and, since every half-loop is embeddable in a loop, ${ }^{4}$ it follows that every half-loop is a subset of some loop.

By a homomorphism $\beta$ of a half-loop $J$, we mean a single-valued

Presented to the Society, April 26, 1947; received by the editors August 18, 1947 .

${ }^{1}$ See Baer and Levi [1] and Kurosch [1]. The numbers in brackets refer to the bibliography at the end of this paper.

${ }^{2}$ Bruck pointed out the fact that a characteristic subloop need not be normal. See Bruck [1].

${ }^{3}$ See Bates [1].

4 See Bates [1]. 
mapping of $J$ into some loop $T$ such that if $x+y$ is in $J$, then $(x+y) \beta$ $=x \beta+y \beta$ in $T$. An isomorphism $\alpha$ of the half-loop $J$ upon the halfloop $K$ is a homomorphism of $J$ upon $K$ which is one-to-one and for which $\alpha^{-1}$ is a homomorphism of $K$ upon $J$.

The half-loop $J$ is closed in the half-loop $K$ if $J \subset K$ and if, given any two of the elements $a, b, c$ in $J$ with $a+b=c$ in $K$, the third element is in $J$. We may then define generation as follows: The halfloop $K$ is generated by its sub-half-loop $J$ if no proper sub-half-loop of $K$ containing $J$ is closed in $K$.

The half-loop $K$ is free over its sub-half-loop $J$ if every homomorphism of $J$ into any loop $T$ may be extended to a homomorphism of $K$ into the same loop $T$. In particular, a free loop $F$ is one which is freely generated by a subset $J$ (called a free basis of $F$ ) having the property: $a+b=c$ in $J$ implies that at least one of the elements $a, b$ is 0 . The cardinal number of nonzero elements in a free basis of the free loop $F$ is called the rank of $F$. A free cyclic loop is a free loop of rank one.

The loop $L$ is the free sum of its subloops $L(v)$ (we write $\left.L=\sum^{*} L(v)\right)$, if $L(v) \cap L(w)=0$ for $v \neq w$, and if $L$ is freely generated by $U L(v)$. A free loop, then, is the free sum of free cyclic loops.

In [I] it is proved that if $J$ is any half-loop, there exists one and essentially only one loop $L$ which is freely generated by $J$, so that the existence of free loops and of free sums of loops is assured. In the appendix of [I] it is also shown that the above definitions are (relatively) analogous to the usual definitions of free groups and free sums of groups.

The following statements, here numbered for future reference, either follow immediately from the statements made above or have been proved in [I].

$1^{0}$. If the loop $L$ is freely generated by $J$, if the loop $T$ is freely generated by $K$, and if there exists an isomorphism $\alpha$ of $J$ upon $K$, then there exists one and only one isomorphism of $L$ upon $T$ which induces $\alpha$ in $J$.

$2^{0}$. If $J C K$ are two sub-half-loops of the loop $L$,

(a) If $L$ is freely generated by $K$ and if $K$ is freely generated by $J$, then $L$ is freely generated by $J$, and:

(b) If $L$ is freely generated by $J$ and if $K$ is generated by $J$, then $L$ is freely generated by $K$.

$3^{0}$. If $L=\sum^{*} L(v)$, then $L=L(w) * \sum_{v \neq w}^{*} L(v)$.

$4^{0}$. If $L=A * B$, and if there exists an isomorphism $\alpha$ of $L$ upon the loop $T$, then $T=A \alpha * B \alpha$. 
Since our definition of free sum of loops is not a constructive one we need the following lemma: 5

LEMma A. If $L=A * B, A, B \neq 0$, then:

(a) If $0 \neq x, y$ are elements of $A \cup B$ for which $x+y=y+x$ in $L$, then $x, y$ are both in $A$ or $x, y$ are both in $B$.

(b) If $0 \neq x, y, z$ are elements of $A \cup B$ for which $(x+y)+z=x$ $+(y+z)$ in $L$, then $x, y, z$ are all in $A$, or $x, y, z$ are all in $B$.

Proof. (a) Suppose that $0 \neq x, y$ are elements of $A \cup B$ for which $x+y=y+x$ in $L$. Assume that $x, y$ are not both in $A$ and are not both in $B$. Then $x$ is in $A, y$ in $B$, or $x$ is in $B, y$ in $A$. Since these two cases are symmetric, we consider only the first case.

Since $A \cap B=0, x+y$ and $y+x$ are undefined in $A \cup B$. Consider the system $S$ composed of elements in $A \cup B$ together with symbols $x \circ y, y \circ x$, with $x \circ y \neq y \circ x$. Define a rule of composition, + , in $S$ as follows:

If $s, s^{\prime}$ are in $A$, let $s+s^{\prime}$ be the uniquely defined sum of $\left(s, s^{\prime}\right)$ in $A$.

If $s, s^{\prime}$ are in $B$ let $s+s^{\prime}$ be the uniquely defined sum of $\left(s, s^{\prime}\right)$ in $B$.

For the given elements $x, y$, define $x+y=x \circ y$ and $y+x=y \circ x$.

Furthermore, we define: $(x+y)+0=x+y=0+(x+y)$ and $(y+x)$ $+0=y+x=0+(y+x)$.

We require the above to be the only sums defined in $S$. In particular, then, $a+b$ for $0 \neq a$ in $A, 0 \neq b$ in $B$, is defined only for $a=x, b=y$; and $b+a$, for $0 \neq b$ in $B, 0 \neq a$ in $A$, is defined only for $b=y$ and $a=x$.

Then $S$ is a half-loop under this composition and there exists one and essentially only one loop $L^{\prime}$ which is freely generated by $S$. But then $L^{\prime}$ is generated by $A \cup B$ since the subloop of $L^{\prime}$ which contains $A \cup B$ must contain $S$ (since it must contain $x+y$ and $y+x$ ). Furthermore, if $\alpha$ is any homomorphism of $A \cup B$ into a loop $T$, then in $T$ there exists one and only one $z^{\prime}$, and one and only one $w^{\prime}$, such that $x \alpha+y \alpha=z^{\prime}$ and $y \alpha+x \alpha=w^{\prime}$, so that $\alpha$ may be extended to a homomorphism $\beta$ of $S$ by mapping $x+y$ on $z^{\prime}$ and $y+x$ on $w^{\prime}$. Since $L^{\prime}$ is free over $S$, there exists one and only one homomorphism $\gamma$ of $L^{\prime}$ inducing $\beta$ in $S$ and $\alpha$ in $A \cup B$-that is, $\alpha$ may be extended to a homomorphism of $L^{\prime}$ into $T$ and hence $L^{\prime}$ is free over $A \cup B$.

But $L$ is freely generated by $A \cup B$, since $L=A * B$. Hence (by $1^{\circ}$ ), there exists an $(A \cup B)$-isomorphism of $L$ upon $L^{\prime}$ and therefore $x+y \neq y+x$ in $L$. But this is a contradiction since we chose $x, y$ in $A \cup B$ such that $x+y=y+x$ in $L$. Hence our assumption that both

' It is fairly obvious that a much more general proposition than Lemma $A$ is valid but as its statement would be somewhat tedious the simpler form sufficient for the purposes of this paper is here preferred. 
$x, y$ were not in $A$ and not in $B$ is false, proving (a).

(b) Given $0 \neq x, y, z$ in $A \cup B$, with $(x+y)+z=x+(y+z)$ in $L$, suppose that $x, y, z$ are not all in $A$ and are not all in $B$. Then there are only two nonsymmetric possibilities: (1) $x, y$ in $A$, but $z$ in $B$, or (2) $x, z$ in $A$, but $y$ in $B$. We discuss these separately:

(1) $x, y$ in $A$; $z$ in $B$; then $x+y=a$, where $a$ is a uniquely defined element in $A$, but $y+z$ and $x+(y+z)$ are undefined in $A \cup B$.

i. If $a=0$, then $a+z=z$. We define a system $S$ consisting of the elements of $A \cup B$, together with distinct symbols $y \circ z$ and $x \circ(y \circ z)$, and define a composition, + , in $S$ as follows: $s+s^{\prime}$ is the uniquely defined sum of $s, s^{\prime}$, when this sum is defined in $A \cup B$ for $s, s^{\prime}$ in $A \cup B ; y+z=y \circ z ; x+(y+z)=x+(y \circ z)=x \circ(y \circ z) ;$ and $s+0$ $=0+s=s$ for all $s$ in $S$. Then $S$ is a half-loop and the argument from here on goes through as in part (a).

ii. If $a \neq 0$, then $a+z$ is also undefined in $A \cup B$ and we adjoin to $S$ in $\mathrm{i}$ a new symbol, $a \circ z$, and define in the extended system one more sum, $a+z=a \circ z$, before completing the argument.

(2) $x, z$ in $A$, but $y$ in $B$; then $x+y, y+z, x+(y+z)$, and $(x+y)+z$ are all undefined in $A \cup B$. Hence, as before, constructing a system $S$ to include the elements of $A \cup B$, we adjoin now four distinct symbols, $x \circ y, y \circ z, x \circ(y \circ z)$, and $(x \circ y) \circ z$, and define an operation, + , in $S$ as follows: For $s, s^{\prime}$ in $A \cup B$, with a (unique) sum defined in $A \cup B$ (hence $s, s^{\prime}$ both in $A$ or both in $B$ ), we define $s+s^{\prime}$ to be this sum; we define, for the given $x, y, z$, the following sums: $x+y=x \circ y$, $y+z=y \circ z,(x+y)+z=(x \circ y)+z=(x \circ y) \circ z$, and $x+(y+z)=x$ $+(y \circ z)=x \circ(y \circ z)$. We also require that $0+s=s+0=s$, for every $s$ in $S$. Then $S$ is a half-loop, as is easily verified, and the argument proceeds as before.

We define a normal subloop of the loop $L$ to be one which is the kernel of some homomorphism of $L$. We may now prove:

THEOREM 1. A free summand of a loop $L$ is normal if and only if the free summand is 0 or $L$ (that is, nontrivial free summands are not normal).

Proof. Let $L=A * B$ (by $3^{0}$ this is no loss in generality). If $A$ (or $B$ ) is 0 , then $B$ (or $A$ ) is equal to $L$ and hence $A$ and $B$ are both (trivial) normal subloops of $L$.

Suppose that $B$ is a normal subloop of $L$. Then there exists a homomorphism $\alpha$ of $L$ into a loop $T$ such that $B$ is the kernel of $\alpha$. If $B=L$ or if $B=0$, the theorem is proved. Hence suppose that $B \neq 0$, $B \neq L$. Then $A \neq 0$ and there exists $a \neq 0$ in $A$ and $b \neq 0$ in $B$. Let $a^{\prime}$ be 
the unique solution $x$ in $A$ to the equation $x+a=0$, and consider the element $c=a^{\prime}+(a+b)$ in $L$.

Suppose that $c=a^{\prime}+(a+b)$ is in $B$. Since $L=A * B$, there exists one (and only one) endomorphism $\beta$ of $L$ which is the extension of the identity map in $B$ together with the null homomorphism of $A$. Then $c=c \beta=\left[a^{\prime}+(a+b)\right] \beta=a^{\prime} \beta+(a \beta+b \beta)=b \beta=b$. That is, if $c$ is in $B$, then $c=a^{\prime}+(a+b)=b$. But this is impossible since then $a^{\prime}+(a+b)$ $=b=\left(a^{\prime}+a\right)+b$, which, by Lemma $\mathrm{A}$, implies that $a, a^{\prime}, b$, are all in $A$ or all in $B$. Hence $c$ is not in $B$.

But, under the homomorphism $\alpha$ of $L$ into $T$, we have $c \alpha$ $=\left[a^{\prime}+(a+b)\right] \alpha=a^{\prime} \alpha+(a \alpha+b \alpha)=a^{\prime} \alpha+a \alpha=\left(a^{\prime}+a\right) \alpha=0$, so that $c$ is in the kernel of $\alpha$.

Hence $B$ is not normal in $L$ if $B \neq 0, B \neq L$, and similarly, $A$ is not normal in $L$ if $A \neq 0, A \neq L$, proving the theorem.

Lemma B. If $L=F * G$ is a nontrivial decomposition of the loop $L$ into free summands $F, G$, where $F$ is a cyclic free loop on generator $f$, then for any nonzero element $g$ in $G, L=Z * G$, where $Z$ is the cyclic (free) subloop of $L$ generated by $z=f+g$.

Proof. $L$ is generated by the half-loop, $z \cup G$, since the subloop of $L$ which is generated by $z$ and $G$ contains $f+g$ and $G$, and therefore contains $f$ and $G$.

Let $\beta$ be a homomorphism of $z \cup G$ into a loop $T$. In $T$ there exists one and only one element $f^{\prime}$ such that $f^{\prime}+g \beta=z \beta$. We define a mapping $\alpha$ of $f \cup G$ into $T$ as follows: Let $g \alpha=g \beta$ for every $g$ in $G$, and define $f \alpha=f^{\prime}$. This map is well-defined and is a homomorphism of $f \cup G$ into $T$. Since $L$ is freely generated by $f \cup G, \alpha$ may be extended to a homomorphism $\gamma$ of $L$ into $T$. Then $z \gamma=(f+g) \gamma=f \gamma+g \gamma=f \alpha+g \alpha$ $=f^{\prime}+g \beta=z \beta$. Hence $\gamma$ induces $\beta$ on $z$. That is, the homomorphism $\beta$ of $z \cup G$ into $T$ has been extended to a homomorphism of $L$ into $T$. Hence $L$ is freely generated by $z \cup G$ and, therefore (by $2^{\circ}$ ), by $Z \cup G$. That is, $L=Z * G$.

We restate next a theorem proved in [I].

General Subloop Theorem for subloops of Free sums. If $L=\sum^{*} L(v)$, and if $S$ is a subloop of $L$, then $S=F_{S} * \sum^{*}(S \cap L(v))$, where:

(i) $F_{S}$ is a free loop whose rank is uniquely determined by $S$ and the $L(v)$.

(ii) $F_{S}$ has a free basis, $F_{0}$, with the property: If $\alpha$ is an automorphism of $L$ such that $S \alpha=S$ and $L(v) \alpha=L(v)$ for every $v$, then $F_{S} \alpha=F_{S}$ and $\alpha$ effects a permutation of the elements of $F_{0}$. 
A corollary of this theorem is the statement that every subloop of $a$ free loop is free.

Note. If $L$ has a free summand, $F \neq 0$, then $F$ is a free loop if and only if in every decomposition of $L$ into free summands there is a free summand which has as a free summand a free loop $\neq 0$. For, suppose $L=A * B$ is any decomposition of $L$ into free summands. Then $L=F * G$ is also a decomposition of $L$ and $G \neq L$, since $F \neq 0$. By the subloop theorem, $A=F_{A} *(A \cap F) *(A \cap G)$ and $B=F_{B} *(B \cap F)$ * $(B \cap G)$. Now if neither $A$ nor $B$ has a free loop not equal to 0 as a free summand, then $F_{A}=A \cap F=F_{B}=B \cap F=0$, since all these are free loops. But then $A=A \cap G$ and $B=B \cap G$, from which we see that $G \supset A \cup B$. Since $L$ is (freely) generated by $A \cup B$, then $L=G$, which is a contradiction.

THEOREM 2. If $L=A * B, A, B \neq 0$, then $A$ is a characteristic subloop of $L$ if and only if both the following conditions are satisfied:

(i) No free summand not equal to 0 of $A$ is a free loop.

(ii) No free summand not equal to 0 of $A$ is isomorphic to a free summand of $B$.

Proof. Suppose first that $A$ is a characteristic subloop of $L$. We prove first the necessity of (i).

Assume that $A$ has a free summand $\neq 0$ which is a free loop. Then we may represent $A$ as $A=F * A_{1}$, where $F$ is a free cyclic loop generated by $f \neq 0$ (see $\left.3^{0}\right)$. Then $L=\left(F * A_{1}\right) * B=F *\left(A_{1} * B\right)$. Let $b$ be any nonzero element in $B$ and consider the unique element $z$ of $L$ satisfying $z=f+b$. Then $z$ is not in $A$ and not in $B$, since $A \cap B=0$. By Lemma $\mathrm{B}$, we have $L=Z *\left(A_{1} * B\right)$, where $Z$ is the cyclic subloop of $L$ generated by $z$.

We define a mapping $\beta$ of $f \cup\left(A_{1} * B\right)$ upon $z \cup\left(A_{1} * B\right)$ as follows: Let $x \beta=x$ for every $x$ in $A_{1} * B$, and let $f \beta=z$. Then $\beta$ is single-valued since $f$ is not in $A_{1} * B$. Furthermore, $\beta$ is an isomorphism of $f \cup\left(A_{1} * B\right)$ upon $z \cup\left(A_{1} * B\right)$. Since $L$ is freely generated by $f \cup\left(A_{1} * B\right), \beta$ may be extended to an endomorphism $\alpha$ of $L$, but this endomorphism is actually an automorphism of $L$ since $L$ is also freely generated by $z \cup\left(A_{1} * B\right)$ (see $\left.1^{0}\right)$. Since $\alpha$ is an extension of $\beta, F \alpha=Z$. But $A \alpha=\left(F * A_{1}\right) \alpha=F \alpha * A_{1} \alpha=F \alpha * A_{1} \beta=Z * A_{1}$, and hence $A \alpha \neq A$, since $z$ is in $Z * A_{1}$, but $z$ is not in $A$. This is impossible, however, since $A$ is a characteristic subloop of $L$. Hence $A$ can have no free summand $\neq 0$ which is free, proving the necessity of condition (i).

If $A$ has a free summand not equal to 0 which is isomorphic to a free summand of $B$, we may represent $A$ and $B$ as follows: $A=A_{1} * A_{2}$; $B=B_{1} * B_{2}$ with $\beta$ the given isomorphism of $A_{2}$ upon $B_{2}$. We then de- 
fine a map $\alpha$ of $A_{1} \cup A_{2} \cup B_{1} \cup B_{2}=J$ into itself as follows: $x \alpha=x$ for every $x$ in $A_{1} \cup B_{1} ; A_{2} \alpha=A_{2} \beta=B_{2}$; and $B_{2} \alpha=B_{2} \beta^{-1}=A_{2}$. Then $\alpha$ is an automorphism of $J$ and since $L$ is freely generated by $J$, there is one and only one automorphism $\gamma$ of $L$ inducing $\alpha$ in $J$. But $A \gamma=\left(A_{1} * A_{2}\right) \gamma=A_{1} \gamma * A_{2} \gamma$ (by $\left.4^{0}\right)=A_{1} \alpha * A_{2} \alpha=A_{1} * B_{2}$, and since $A \cap B=0, A_{1} * B_{2} \neq A$. Hence $A \gamma \neq A$, in contradiction to the fact that $A$ is a characteristic subloop of $L$. That is, $A$ can have no free summand not equal to 0 which is isomorphic to a free summand of $B$, which finishes the proof of the necessity of conditions (i) and (ii).

Now, assuming that conditions (i) and (ii) are satisfied, let $\alpha$ be any automorphism of $L$. Then $L=A * B=A \alpha * B \alpha$ (by $4^{0}$ ) and $A \alpha$ is isomorphic to $A, B \alpha$ is isomorphic to $B$. Using the Subloop Theorem we have: $A=(A \cap A \alpha) *(A \cap B \alpha)$, since by (i), $A$ has no free summand not equal to 0 which is free. Also, $B \alpha=F_{B \alpha} *(B \alpha \cap A) *(B \alpha \cap B)$. But then $B \alpha \cap A$, a free summand of $A$, is isomorphic to a free summand of $B$ and, by condition (ii), then $B \alpha \cap A=0$ and $A=A \cap A \alpha$.

We have, similarly, $B=F_{B} *(B \cap A \alpha) *(B \cap B \alpha)$ and $A \alpha=(A \alpha \cap A)$ * $(A \alpha \cap B)$, since $A \alpha$ is isomorphic to $A$ and $A$ satisfies condition (i). Then $A \alpha \cap B$, a free summand of $B$, is isomorphic to a free summand of $A$. Hence $A \alpha \cap B=0$, and we have $A \alpha=A \cap A \alpha=A$, proving that $A$ is characteristic.

Corollary. If $L=\sum^{*} L(v)$ (each $L(v) \neq 0$ ) is a nontrivial decomposition of $L$ into free summands, then the $L(v)$ are characteristic subloops of $L$ if and only if no $L(v)$ has a free summand not equal to 0 which is free or which is isomorphic to a free summand of $L(w)$, for $w \neq v$.

Proof. The necessity of the above conditions is an immediate consequence of Theorem 2 , since, for each $L(v), L$ may be represented in the form $L=L(v) * \sum_{w \neq 0}^{*} L(w)$.

Suppose that the conditions of the corollary are satisfied for every $v$. Let $\sum_{w \neq 0}^{*} L(w)=B$, for some fixed $v$; then $L=L(v) * B$. To apply Theorem 2 we must show that no free summand not equal to 0 of $L(v)$ is isomorphic to a free summand of $B$.

Assume that $B=B_{1} * B_{2} ; L(v)=V_{1} * V_{2}$, with $\alpha$ an isomorphism such that $B_{2} \alpha=V_{2}\left(B_{2} \neq 0\right)$. Since $B_{2} \subset B=\sum_{w \neq 0}^{*} L(w)$, then, $B_{2}=\sum_{w \neq 0}^{*}\left(B_{2} \cap L(w)\right)$, using the subloop theorem and the fact that $B_{2}$ contains no free summand not equal to 0 which is a free loop, since $B_{2}$ is isomorphic to $V_{2}$, a free summand of $L(v)$.

Then $V_{2}=B_{2} \alpha=\sum_{w \neq 0}^{*}\left(B_{2} \cap L(w)\right) \alpha$ and hence $B_{2} \cap L(w)$ is isomorphic to a free summand of $V_{2}$ and therefore of $L(v)$. But $L(w) \subset B$ $=B_{1} * B_{2}$ and hence $L(w)=\left(L(w) \cap B_{1}\right) *\left(L(w) \cap B_{2}\right)$ so that 
$B_{2} \cap L(w)$ is also a free summand of $L(w), w \neq v$. This is impossible according to the hypotheses of our corollary and hence the assumption that a free summand not equal to 0 of $L(v)$ is isomorphic to a free summand of $B=\sum_{w \neq v}^{*} L(w)$ is false. Hence we have, for the decomposition of $L=L(v) * B$, the two conditions of Theorem 2 satisfied for $L(v)$, proving that $L(v)$ is a characteristic subloop of $L$.

THEOREM 3. If $L=\sum^{*} L(v)$ is a decomposition of $L$ into characteristic free summands, and if $S$ is a characteristic subloop of $L$, then $S=F * \sum *(S \cap L(v))$, where

(1) $F$ is a free characteristic subloop of $L$.

(2) For every $v, S \cap L(v)$ is a characteristic subloop of $L$ and of $L(v)$.

(3) There exists a free basis, $B$, of $F$ such that every automorphism of $L$ induces $a$ permutation in $B$.

The proof of this theorem is immediate upon application of the subloop theorem, since the $L(v)$ and $S$ are characteristic in $L$.

THEOREM 4. If $L=\sum^{*} L(v)=\sum^{*} K(w)$ are two decompositions of $L$ into characteristic free summands, then $L=\sum_{v, v}^{*}(L(v) \cap K(w))$, where the $L(v) \cap K(w)$ are characteristic in $L$.

Proof. By Theorem 3 , since $L(v)$ is a characteristic subloop of $L=\sum^{*} K(w)$, we have, for each $v, L(v)=\sum_{w}^{*}(K(w) \cap L(v))$, since $L(v)$ has no free summand not equal to 0 which is a free loop. Also, for each $w, K(w) \cap L(v)$ is a characteristic subloop $L=\sum^{*} L(v)$ $=\sum_{v, v 0}^{*}(K(w) \bigcap L(v))$.

THEOREM 5. If $L$ is the free sum of subloops $L(v)$, then there exists one and only one decomposition of $L$ into a free sum of characteristic subloops $K(w)$ such that: (a) every $K(w)$ is a free sum of some of the $L(v)$; (b) No refinement of the decomposition into the $K(w)$ has this property.

Proof. There exists one decomposition of $L$ into characteristic free summands satisfying condition (a)-namely, the trivial one, $L=L$. Consider the cross-cuts of all free summands from any one decomposition of $L$ into characteristic free summands satisfying (a), with each of those of every other such decomposition of $L$. By Theorem 4, then, $L$ is the free sum of all such cross-cuts, the latter being characteristic subloops of $L$. Clearly this last decomposition of $L$ satisfies also (b), proving the theorem.

A loop $L$ is free irreducible if $L$ has no decomposition into nonzero free summands other than the trivial decomposition, $L=L$. In particular, a free loop $F \neq 0$ is free irreducible if (and only if) it is cyclic. For, if $F=A * B$, then $A, B$ are free and $1=\operatorname{rank} F=\operatorname{rank} A+\operatorname{rank} B$.

If the loop $L$ has a decomposition into free irreducible summands 
$L(v)$, none of which is a free loop, it follows immediately from the subloop theorem that such a decomposition is unique and any automorphism of $L$ effects merely a permutation of the $L(v)$. Hence we have a stronger theorem in this case:

TheOREM 6. If $L=\sum^{*} L(v)$ is a nontrivial decomposition of the loop $L$ into free irreducible summands, then $L$ has a nontrivial decomposition into characteristic free summands $K(w)$ if and only if the following two conditions are satisfied: (i) no $L(v)$ is a free loop and (ii) not all the $L(v)$ are isomorphic. This decomposition into the $K(w)$ will of necessity be such that each $K(w)$ is the free sum of some of the $L(v)$ and may be so carried out that no further refinement of the decomposition will yield a characteristic free decomposition of $L$-it will then be unique.

PROof. Suppose $L$ has a nontrivial decomposition into characteristic free summands, $K(w)$. Then no $K(w)$ has a free summand not equal to 0 which is free or is isomorphic to a free summand of $K\left(w^{\prime}\right)$, for $w \neq w^{\prime}$ (Corollary to Theorem 2). It follows from the remarks made above, then, that no $L(v)$ is a free loop and that not all the $L(v)$ are isomorphic.

Conversely, if conditions (i) and (ii) are satisfied, then $L$ obviously has one nontrivial decomposition into characteristic free summands. For, there exists at least one pair of free summands, $L\left(v^{\prime}\right)$ and $L\left(v^{\prime \prime}\right)$, which are not isomorphic. Then $L=A * B$, where $A$ is the free sum of all the $L(v)$ which are isomorphic to $L\left(v^{\prime}\right)$ and $B$ is the free sum of the remaining $L(v)$, is such a decomposition. The decomposition consisting of the cross-cuts of all free summands from any one such decomposition with each of those of every other such decomposition will have the further properties of the theorem.

\section{BIBLIOGRAPHY}

R. BAER AND F. LEVI

1. Freie Produkte und ihre Untergruppen, Compositio Math. vol. 3 (1936) pp. 391398.

G. E. BATES

1. Free loops and nets and their generalizations, Amer. J. Math. vol. 69 (1947) pp. 499-550.

R. BRUCK

1. Simple quasigroups, Bull. Amer. Math. Soc. vol. 50 (1944) pp. 769-781.

2. Contributions to the theory of loops, Trans. Amer. Math. Soc. vol. 60 (1946) pp. 245-354.

A. KUROSCH

1. Über freie Produkte von Gruppen, Math. Ann. vol. 108 (1933) pp. 26-36.

Mount Holyoke College 